Notfall Rettungsmed 2015 · 18:528 DOI 10.1007/s10049-015-0045-5

Online publiziert: 11. August 2015

(c) Springer-Verlag Berlin Heidelberg 2015
H. Dormann

Chefarzt Zentrale Notaufnahme, Klinikum Fürth, Fürth, Deutschland

\title{
MRT der gesamten Wirbelsäule notwendig - nicht nur auf die Schmerzregion allein reduziert
}

\author{
Erwiderung \\ Zum Leserbrief von J.D. Müller-Broich (2015) \\ Transthorakale Echokardiographie und \\ MRT allein der HWS nicht ausreichend. DOI \\ 10.1007/s10049-015-0044-6
}

Sehr geehrter Herr Kollege,

vielen Dank für Ihre fundierten und ergänzenden Kommentare mit dem Fokus der weiterführenden Diagnostik bei genanntem Patienten. Unser Ziel war es aufgrund der häufig verzögerten Diagnostik auf die oft unspezifische Symptomkonstellation im Notaufnahmesetting aufmerksam zu machen, um diesen „blinden Fleck“ in der möglichen Differenzialdiagnostik einer zeitkritischen und schweren Erkrankung zu schließen. Das Hinzuziehen eines Wirbelsäulenchirurgen bei jedem Patienten mit Nackenschmerzen wird wohl in der klinischen Routine so nicht umsetzbar sein. Im Falle eines Initialverdachts muss dann wie von Ihnen gefordert eine MRT der gesamten Wirbelsäule (holospinal) durchgeführt werden und sollte keinesfalls auf die Schmerzregion alleine reduziert werden. Die Hinweise zur erweiterten Fokussuche sind wertvoll, lassen sich aber leider nicht evidenzbasiert nachvollziehen, sondern bedürfen konkreter klinischer Hinweise im Einzelfall. Die Verlegung in die Neurochirugie wurde in diesem Fall so veranlasst und steht im Allgemeinen stellvertretend für eine Abteilung mit geeigneter operativer und auch konservativer Kompetenz im Bereich der Wirbelsäulenerkrankungen, was unsererseits leider so nicht benannt wurde.

Ihrer Forderung nach SOP kann ich nur unterstützen, um so eine raschere und frühzeitigere Diagnostik und damit auch
Therapieinitiierung zu ermöglichen. Als Vorstandsmitglied der Deutschen Gesellschaft für interdisziplinäre Notfall- und Akutmedizin (DGINA e. V.) kann ich hierzu der Deutschen Wirbelsäulengesellschaft nur meine Bereitschaft zur Zusammenarbeit signalisieren.

Ich danke Ihnen für die wertvollen Ergänzungen.

Mit freundlichen Grüßen

Harald Dormann

\section{Korrespondenzadresse}

Prof. Dr. med. H. Dormann

Chefarzt Zentrale Notaufnahme

Klinikum Fürth

Jakob Henle Str. 1

90766 Fürth

harald.dormann@klinikum-fuerth.de 